\title{
On the indices of plant-plant competition and their pitfalls
}

\author{
Lauri Oksanen, Marek Sammul and Merike Mägi
}

Oksanen, L., Sammul, M. and Mägi, M. 2006. On the indices of plant-plant competition and their pitfalls. - Oikos 112: 149-155.

\begin{abstract}
The index of relative competition intensity (RCI) has serious built-in biases, due to its asymptotic behavior when competition intensity is high and its tendency to obtain very low values when plants with neighbors intact perform better than neighbor removal plants. These biases have been partially corrected in the index of relative neighbor effect (RNE), but the existence of fixed upper and lower bounds $(-1 \leq \mathrm{RNE} \leq+1)$ still creates problems and biases in communities where the average intensity of competition or facilitation is high and plant performance pronouncedly varies in space. The third commonly used index, the logarithm of response ratio (lnRR), is mathematically and statistically sound, but when computed from pair-wise comparisons between neighbor removal and control plants, this index reflects the geometric mean of the treatment effect. Moreover, linear patterns in lnRR reflect exponential patterns in the intensity of competition. As the interest of ecologists usually focuses on arithmetic means, we propose a corrected index of relative competition intensity, CRCI $=\operatorname{arc} \sin (\mathrm{RNE})$. This index is fairly linear within the observed ranges of competition and facilitation, and for the range of competition intensities where RNE behaves reasonably, the two indices obtain almost identical values.

We compared the performance of the four indices, using both imagined and real data, the latter from systems where the responses of plants to neighbor removal ranged from weak to moderate, so that RNE and CRCI were expected to behave similarly. The indices were computed both from pooled data for each community and as averages of pair-wise comparisons. $\operatorname{lnRR}$ and CRCI were found to behave in a consistent and biasfree manner, yielding similar results regardless of method of computation. This was, by and large, the case with RNE, too, but as the values of indices grew, the values from pair-wise comparisons became increasingly smaller than values computed from pooled data. RCI yielded grossly aberrant results in computations based on pair-wise comparisons. Therefore, the further use of RCI is unadvisable and studies where RCI has been derived from pair-wise comparisons should be excluded from meta-analyses.
\end{abstract}

L. Oksanen, Dept of Ecology and Environmental Science, Umeå Univ., SE-901 87 Umeå, Sweden. Present address: Dept of Biology, Univ. of Turku, FIN-20014 Turku, Finland (lauoks@utn.fi). - M. Sammul, Inst. of Agricultural and Environmental Sciences, Estonian Agricultural Univ., Riia 181, EE-51014 Tartu, Estonia. - M. Mägi, Inst. of Botany and Ecology, Univ. of Tartu, Lai 40, EE-51005 Tartu, Estonia.

\section{Introduction}

A straightforward way to estimate the intensity of competition or facilitation in plant communities is to randomly choose individuals of medium-sized plant species, to remove their neighbors, and to compare the performances of thus manipulated and control plants. In comparisons between different communities, the responses of plants must be corrected for the trivial impacts of differences in productivity of the site (Aarssen and Epp 1990, Grace 1995). Two approaches for doing this have been developed. First, the differences between the performances of manipulated plants and control plants have been related to favorability of the site by dividing the difference between the performance parameters of manipulations and controls 
by the performance of the manipulated plants, which yields the index of relative competition intensity (RCI, Goldberg and Scheiner 1993, Wilson and Tilman 1993, Grace 1995), or by the parameter value of the treatment that performs better (relative neighbor effect, RNE, Markham and Chanway 1996, Callaway et al. 2002). Alternatively, the logarithm of the performance ratio of manipulated plants to controls has been used ( $\ln R R$, Cahill 1999, 2002, Goldberg et al. 1999, Hedges et al. 1999, Gough et al. 2001).

While both approaches erase the trivial effects of productivity on the index of competition intensity, they introduce other problems, which have obtained little attention, and which become especially pronounced if index values are derived from pair-wise comparisons to obtain interval estimates for the intensity of competition or facilitation in the focal communities. The basic reason is that RCI and $\ln R R$ are based on upward convex functions, while RNE is bounded near both of its maximum and minimum value $(+1$ and -1 , respectively). Hence, the means of index values derived from pair-wise comparisons may differ systematically from the index value based on pooled data, depending on the variability of the performance of individual plants.

\section{Mathematical interpretation and statistical behavior of currently used competition indices}

The currently used indices for the measurement of intensity of competition are obtained as

$\mathrm{RCI}=\left(\mathrm{X}_{\mathrm{r}}-\mathrm{X}_{\mathrm{c}}\right) / \mathrm{X}_{\mathrm{r}}$

$\mathrm{RNE}=\left(\mathrm{X}_{\mathrm{r}}-\mathrm{X}_{\mathrm{c}}\right) / \max \left(\mathrm{X}_{\mathrm{r}}, \mathrm{X}_{\mathrm{c}}\right)$

$\ln R \mathrm{R}=\ln \left(\mathrm{X}_{\mathrm{r}} / \mathrm{X}_{\mathrm{c}}\right)$

where RCI is the coefficient of relative competition intensity, RNE is the relative neighbor effect, lnRR is the logarithmic response ratio, $\mathrm{X}_{\mathrm{r}}$ is the performance of manipulated plants, whose competitors have been removed and $\mathrm{X}_{\mathrm{c}}$ is the performance of controls, which grow interacting with neighbors. The values for $X_{r}$ and $\mathrm{X}_{\mathrm{c}}$ can be either computed by first pooling the values of individual performance of plants in different treatments for each habitat, to minimize the errors caused by aberrant behavior of individual plants, or by randomly pairing neighbor removal and control plants and calculating the averages of pair-specific indices for each habitat to obtain interval estimates for index values. Mathematical and statistical soundness requires that the expect value of the index must be the same regardless which method is chosen.

As pointed out by Goldberg et al. (1999), the RCI and $\ln R R$ indices are mathematically related, though not in the way proposed by them $(\ln R R \neq-\ln (\mathrm{RCI}-1)$; notice that RCI- $1<0$, and logarithms of negative numbers are not defined!). The correct relationship is: $\ln R \mathrm{R}=\ln (1-\mathrm{RCI})$. In spite of its seeming simplicity, this relationship is quite complex, as there is no way to take a logarithm of a sum without first computing its numerical value. Below, we will see that the two indices are even very differently related to variance in plant performance.

When derived from pair-wise computations, the mean value of $\ln R R$ has a clear mathematical interpretation, deriving from the basic rule that the sum of logarithms equals to the logarithm of the product:

$$
\begin{aligned}
\ln R R & =\Sigma\left(\operatorname{lnR} R_{\mathrm{i}}\right) / \mathrm{n}=\Sigma\left(\ln \left(\mathrm{X}_{\mathrm{ri}} / \mathrm{X}_{\mathrm{ci}}\right)\right) / \mathrm{n} \\
& =\left(\ln \Pi\left(\mathrm{X}_{\mathrm{ri}} / \mathrm{X}_{\mathrm{ci}}\right)\right) / \mathrm{n}
\end{aligned}
$$

where $\ln R R_{i}$ stands for the index values derived from $i$ :th pair-wise comparison, $\mathrm{X}_{\mathrm{ri}}$ is the performance of $\mathrm{i}$ :th manipulated plant in the $\mathrm{i}$ :th pair, $\mathrm{X}_{\mathrm{ci}}$ is the performance of the control plant in this pair, and $n$ is the number of pairs to be compared. The mean value of pair-wise indices thus represents the logarithm of the geometric mean of individual index values. While there is nothing wrong in using geometric means to characterize sets of values, it is important to realize that geometric and arithmetic means are not equivalent. Geometric means are very sensitive to the variability of the data and, especially, to the occurrence of small values in the data set. A single pair where the manipulated plant has failed (i.e. where $\mathrm{X}_{\mathrm{ri}} \approx 0$ ), while the performance parameter of the control is positive, makes the geometric mean approach zero, and its logarithm will be a negative number with very large absolute value, regardless of the outcome of the other pair-wise trials. When the $\operatorname{lnRR}$ index obtains higher value in community $\mathrm{A}$ than in community B we thus cannot infer that competition intensity would, at average, be higher in community A. The correct interpretation is that competition is either more intense or shows less variation in community $\mathrm{A}$.

Moreover, linear patterns in lnRR along environmental gradients reflect exponential patterns in the intensity of plant-plant competition. This distinction is not a mere technicality. Positive exponential patterns imply that the relationship is very strong for very large values and weak or non-existent for much of the data set, while the converse applies for negative exponential patterns. Hence, results obtained at extremes are crucial for linear patterns in $\operatorname{lnRR}$, and the reliability of meta-analyses based on $\ln R R$ are strongly dependent on the reliability of the few most extreme data points.

Logarithms of ratios have the property that if numerators and denominators are interchanged, the signs change but the absolute values remain constant. Thus, if the variance in the performance of control and neighbor removal plants is equal, then the expected value of the $\operatorname{lnRR}$ index is the same, regardless of whether the index is derived from pooled data or from pair-wise comparisons. This is a big "if", because neighbor removal both reduces competition and exposes 
the plant to environmental stress thus influencing the variability of plant performance. Therefore, a researcher using $\ln R R$ should always check whether the data allows for deriving bias-free indices from pair-wise comparisons. We can conclude that the $\operatorname{lnRR}$ index is a mathematically and statistically sound tool for studying exponential patterns in geometric means. However, we have not seen a single paper where an author using the $\operatorname{lnRR}$ index had shown awareness of the consequences of focusing on geometric means and exponential patterns.

The properties of the RCI index are more problematic. Exceptionally poor performance of a single neighbor removal plant has a major impact on the value of RCI, computed from pair-wise comparisons. Equally poor performance of a control plant has practically no impact whatsoever because the behavior of the index is totally asymmetric. The problems with RNE are similar though much less dramatic. In the case of RNE serious and potentially biasing asymmetry emerges if the values obtained from individual trials land to the part of the curve, where it changes from nearly linear to visibly asymptotic. Then, deviations to one direction have much stronger impact on the mean value of RNE than equally large deviations to the other direction.

To illustrate the problems, let us assume that we study plant-plant interactions on a lush meadow, where intense competition prevails. Medium-sized forbs and grasses (typical targets of competition studies) are thus severely suppressed and perform, at average, 1000 times better if their competitors are locally removed. For this community, the values for the two competition indices, derived from pooled data obtained from infinitely large samples, are as follows: $\mathrm{RCI}=\mathrm{RNE}=+0.999$, and $\ln R R=+6.91$. Now assume that we study the intensity of competition using 20 pairs of ramets, randomly assigned to neighbor removals and controls. Suppose that 19 pairs yield results corresponding to the average situation. In one pair, however, the manipulated plant happens to be almost killed by some stress factor, a grazer or a pathogen. Hence, the control plant performs 100 fold better than the manipulated one. For this $\mathrm{i}$ :th pair we obtain $\mathrm{RCI}_{\mathrm{i}}=-99.0, \mathrm{RNE}_{\mathrm{i}}=-0.99$ and $\ln R R_{i}=-4.61$. By averaging from pair-wise comparisons, we obtain following averages for our indices: $\mathrm{RCI}=-4.00, \mathrm{RNE}=+0.90$, and $\ln \mathrm{R} \mathrm{R}=+6.33$.

We see that when computed from pair-wise comparisons, all three indices are fairly sensitive to such occasional reversals in the performance of control and neighbor removal plants, which have practically no impact on index values computed from pooled data. The reason is the upward convexity of the logarithmic and rational functions underlying the indices. The magnitudes of the errors and biases thus created are vastly different. In the case of $\operatorname{lnRR}$, the error is not dramatic, unless the performance parameter of the aberrant plant is very close to zero. Both RCI and
RNE indices become more seriously biased. In the case of RCI, the impact of occasional reversal of plant performance on the index value is positively bizarre. A single reversal can result in a large, negative index value, indicating strong facilitation, even in a community where intense competition was assumed to prevail. For RNE, the impact on mean is less dramatic. However, the standard deviation increases (in this case, sd= 0.44 ), and since the index is bound to the interval $-1 \leq \mathrm{RNE} \leq+1$, such increase in standard deviation easily creates a situation where zero is included in the confidence interval of the mean, even in communities characterized by intense competition or facilitation.

\section{Correcting for the biases of RCI and RNE}

The introduction of the RNE index by Markham and Chanway (1996) was a good beginning in the effort to create a linear index of competition intensity, which allows for bias-free computation of interval estimates. However, the work was left unfinished. While the use of the larger value in the denominator corrects for the bizarre asymmetry of RCI, it does nothing to the insensitivity of the index to differences between high and very high intensity of competition or facilitation. The standard way to correct for problems caused by fixed upper and lower bounds is to use arc sin transformation, which extends the range of arguments where the function behaves nearly linearly. There are several ways of doing this transformation, but in the present context, the simplest one seems appropriate. We thus propose corrected index of relative competition intensity, CRCI, as

$$
\begin{aligned}
\mathrm{CRCI} & =\arcsin \left(\left(\mathrm{X}_{\mathrm{r}}-\mathrm{X}_{\mathrm{c}}\right) /\left(\max \mathrm{X}_{\mathrm{r}}, \mathrm{X}_{\mathrm{c}}\right)\right) \\
& =\arcsin (\mathrm{RNE})
\end{aligned}
$$

Unlike RCI, lnRR and RNE, CRCI behaves practically linearly within a wide range of intensities of competition and facilitation, allowing for detection of linear trends in the intensity of plant-plant competition and making it possible to derive bias-free interval estimates of the intensity of plant-plant interactions.

\section{Do the differences matter: an empirical example}

The practical significance of the problems discussed above was studied by computing $\operatorname{lnRR}$, RCI, RNE and CRCI indices from our experiments concerning the impact of neighbor removal on the performance of Solidago virgaurea in various Estonian and north Norwegian communities. We focused on the question whether the different indices yielded consistent results in between-community comparisons, since this question has been the subject of several meta-analyses and large scale experimental projects (Reader et al. 1994, 
Goldberg et al. 1999, Hedges et al. 1999, Callaway et al. 2002). The communities included in our data base span ranges of primary productivity typically covered in studies of this kind; the difference between the least productive community (an arctic-alpine snow-bed in northern Norway) and the most productive one (a seashore meadow in Estonia) being about sevenfold, when accumulated annual growth is used as the index of primary productivity. In total, the study covered 16 communities (8 in Estonia, 8 in northern Norway). Each community was represented by 14 to 30 Solidago ramets. Half of them were randomly assigned to neighbor removal treatments, the other half being left as controls. The details of the study are reported by Sammul et al. (2005).

As the ramets were not initially paired, we did the pairing necessary for pair-wise computations randomly afterwards. Different randomizations, where each neighbor removal was paired with one control (giving 7 to 15 pairs) turned out to yield rather different results. We continued using 25, 50 and 100 random pairs. Finally, we performed computations of indices by pairing all neighbor removals with all controls and also computed coefficients after first pooling the samples of treatments. The results for the analyses using all possible pairs and
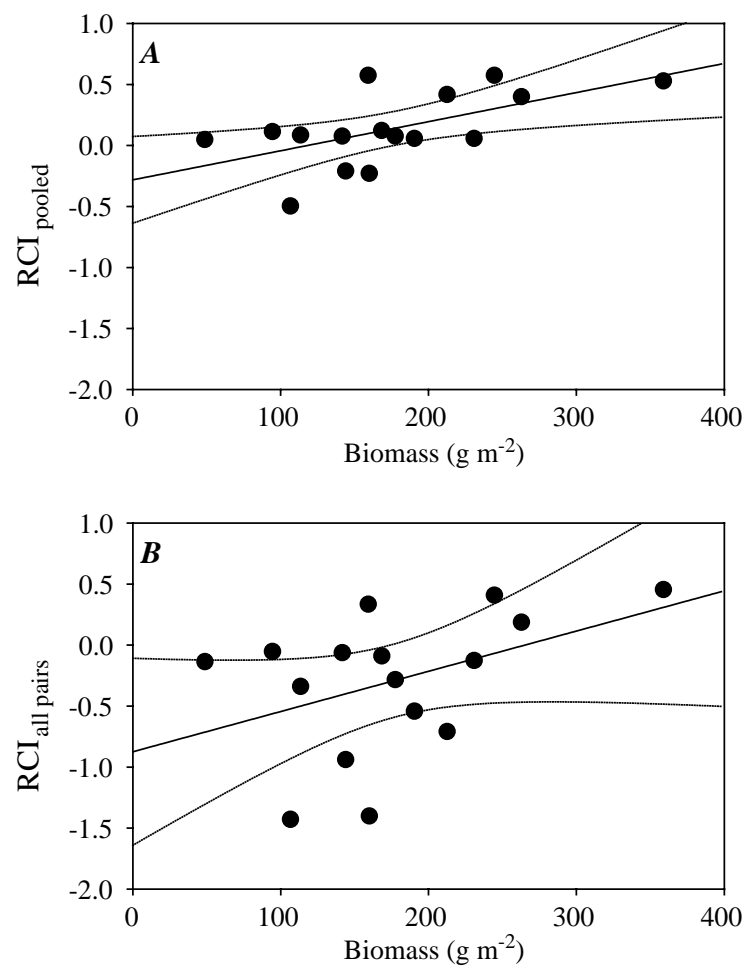

Fig. 1. Relation between community biomass and index of relative competition intensity $(\mathrm{RCI})$, computed from pooled data (A) for each habitat and from pair-wise comparisons (B) between treated plants (all neighbors removed) and control plants.
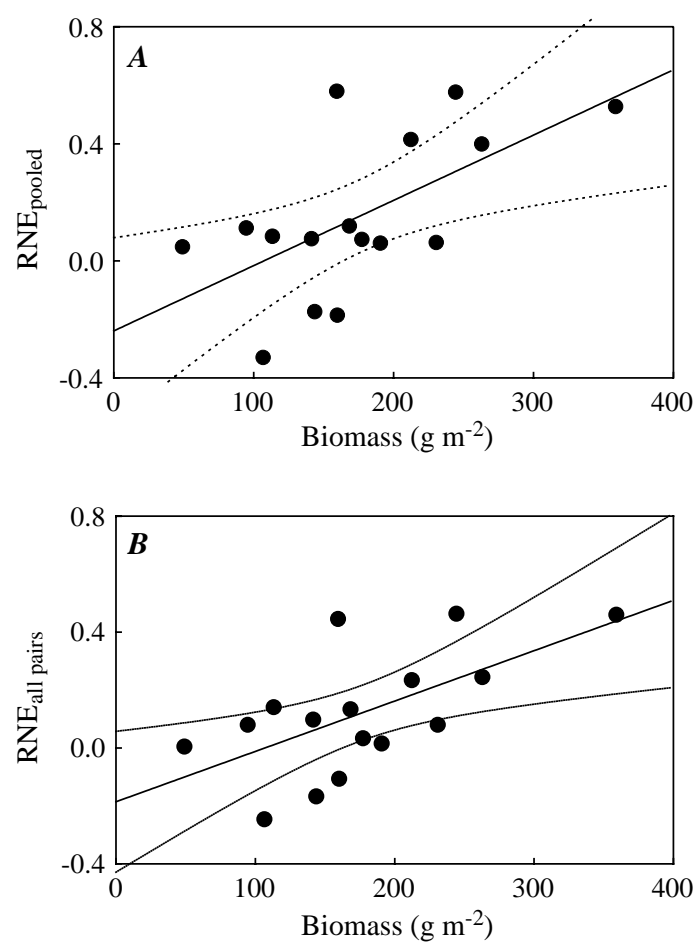

Fig. 2. Relation between community biomass and index of relative neighbour effect (RNE), computed from pooled data (A) for each habitat and from pair-wise comparisons (B) between treated plants (all neighbors removed) and control plants.

pooled values are presented in Fig. 1 (for RCI), Fig. 2 (for RNE), Fig. 3 (for $\operatorname{lnRR}$ ) and Fig. 4 (for CRCI), respectively. In all figures, planes $\mathrm{A}$ refer to analyses based on pooled data and planes B refer to comparisons of all neighbor removals to all controls. The numerical values of the critical parameters (slope, y-intercept, $\mathrm{R}^{2}$ ) are presented in Table 1 for all analyses, along with the two-tailed p-values for the null hypothesis that the slope or respectively the y-intercept is equal to zero. The mean values of coefficients and the two-tailed $p$-values for the null hypothesis that the coefficients' mean is zero, are presented in Table 2.

When based on pooled data (Fig. 1A, 2A, 3A, 4A, Table 1), all methods yield a regression line with a significantly positive slope, and a y-intercept statistically indistinguishable from zero, implying that the intensity of competition increases with increasing primary productivity and neutral plant-plant relationships prevail in totally barren areas. Moreover, when based on pooled data for each community, all methods indicate that competitive plant-plant relationships prevail in northern Europe (Table 2). The message is unambiguous $(\mathrm{p}<0.05)$ for RNE, $\operatorname{lnRR}$ and CRCI, whereas in the case of RCI, the difference of the mean from zero is not statistically significant $(\mathrm{p}=0.088)$. Moreover, all 

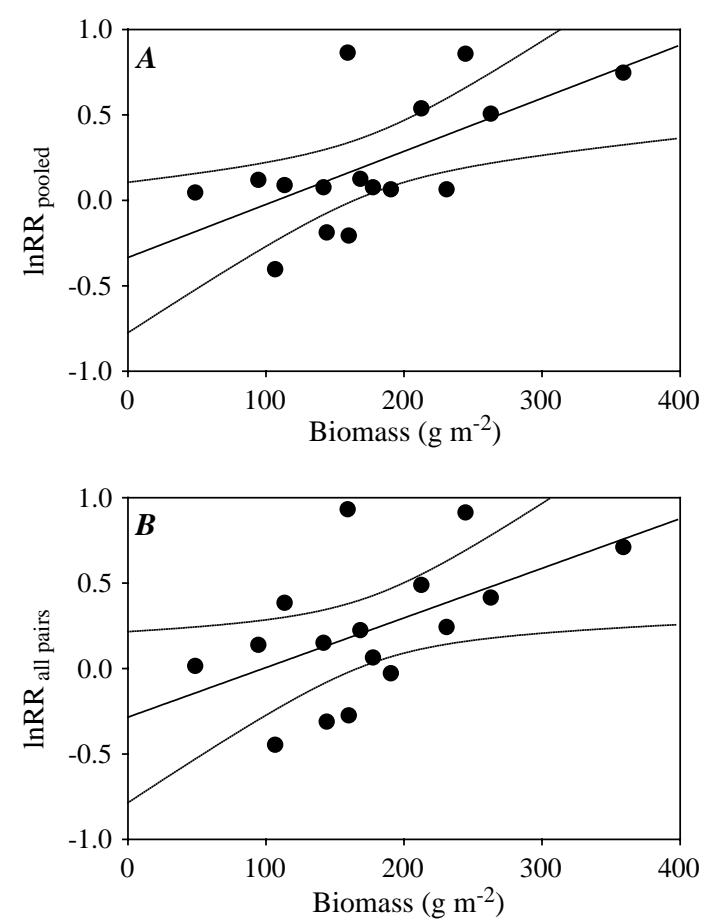

Fig. 3. Relation between community biomass and logarithm of response ratio (LnRR), computed from pooled data (A) for each habitat and from pair-wise comparisons (B) between treated plants (all neighbors removed) and control plants.

methods indicate that the relationship is not linear but step-wise; this pattern has been confirmed by a statistical analysis of residuals (Sammul et al. 2005).

In pair-wise comparisons, the differences between RCI and the other methods become dramatic. When $\operatorname{lnRR}$ or CRCI are used, the message remains the same as obtained by using pooled data (Fig. 2-4, Table 1 and 2). This is largely true for RNE, too, except for three inconsistencies. First, the use of pair-wise comparisons considerably reduces the highest index values (from about 0.6 to about 0.45 , Fig. 2). Second, the p-values for the y-intercept are consistently lower than obtained using $\ln R \mathrm{R}$ or CRCI, being statistically significant in two out of the five cases. Third, the slope of the regression line is consistently lower when RNE was derived from pair-wise comparisons, obviously due to the smaller values of the index at both negative and positive extremes of observed interaction strength.

For RCI the visual pattern and parameter values (Table 1,2 ) change totally when the index is derived from pair-wise comparisons (Fig. 1). The decline in the highest values is similar to the RNE, but the lowest values decline dramatically, from about -0.5 to between -1.0 and -1.5 . Moreover, most trials yield significantly negative y-intercepts, almost significantly negative means $(0.05<\mathrm{p}<0.1)$, whereas the slope of the
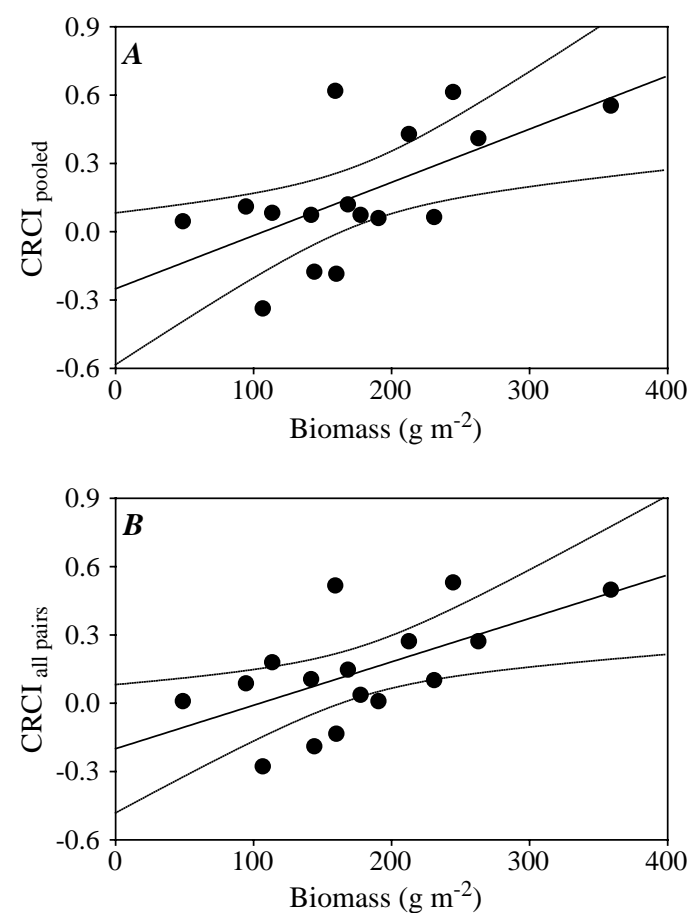

Fig. 4. Relation between community biomass and corrected index of relative competition intensity (CRCI), computed from pooled data (A) for each habitat and from pair-wise comparisons (B) between treated plants (all neighbors removed) and control plants.

regression is not statistically significant. The message of pair-wise computed RCI values is thus that plant-plant relationships in northern Europe are primarily neutral or facilitative, regardless to the productivity of the habitat (Table 1,2). This message is totally inconsistent with the implications of the other indices and with the implications of RCI-based comparisons, when the index is derived from pooled data.

\section{Discussion and conclusions}

Our results indicate that interval estimates of competition intensity obtained with the most widely used competition index - RCI - are strongly influenced by the variability of plant performance, if the index is derived from pair-wise comparisons. Consequently, this index can create entirely false implications concerning patterns in nature. Depending on the distribution of cases where some neighbor removal plants have performed exceptionally poorly, real trends can be masked and phony trends can emerge. Consequently, use of RCI seems unadvisable, and papers where RCI has been derived from pair-wise comparisons should not be included in meta-analyses. 
Table 1. Parameter values and descriptive statistics of regression analyses of different competitive indices against community productivity.

\begin{tabular}{|c|c|c|c|c|c|c|c|c|}
\hline \multirow[t]{2}{*}{ Coefficient } & \multicolumn{3}{|c|}{ Intercept } & \multicolumn{3}{|c|}{ Slope } & \multicolumn{2}{|c|}{ Regression } \\
\hline & value & $t_{14}$ & $\mathrm{p}$ & value & $\mathrm{t}_{14}$ & $\mathrm{p}$ & $\mathrm{R}^{2}$ & $\mathrm{~F}_{1,14}$ \\
\hline $\begin{array}{l}\mathrm{RCI}_{\text {min }} \\
\mathrm{RCI}_{25} \text { pairs } \\
\mathrm{RCI}_{50} \text { pairs } \\
\mathrm{RCI}_{100 \text { pairs }} \\
\mathrm{RCI}_{\text {all pairs }} \\
\mathrm{RCI}_{\text {pooled }}\end{array}$ & $\begin{array}{l}-0.97 \\
-0.62 \\
-0.99 \\
-0.93 \\
-0.87 \\
-0.28\end{array}$ & $\begin{array}{l}-2.26 \\
-1.58 \\
-2.53 \\
-3.21 \\
-2.46 \\
-1.71\end{array}$ & $\begin{array}{l}0.040 \\
0.136 \\
0.024 \\
0.006 \\
0.028 \\
0.110\end{array}$ & $\begin{array}{l}0.0036 \\
0.0021 \\
0.0039 \\
0.0038 \\
0.0033 \\
0.0024\end{array}$ & $\begin{array}{l}1.61 \\
1.00 \\
1.90 \\
2.50 \\
1.76 \\
2.75\end{array}$ & $\begin{array}{l}0.130 \\
0.333 \\
0.079 \\
0.026 \\
0.100 \\
0.016\end{array}$ & $\begin{array}{l}0.16 \\
0.07 \\
0.20 \\
0.31 \\
0.18 \\
0.35\end{array}$ & $\begin{array}{l}2.60 \\
1.01 \\
3.59 \\
6.24 \\
3.11 \\
7.57\end{array}$ \\
\hline $\begin{array}{l}\mathrm{RNE}_{\text {min }} \\
\mathrm{RNE}_{25} \text { pairs } \\
\mathrm{RNE}_{50 \text { pairs }} \\
\mathrm{RNE}_{100 \text { pairs }} \\
\mathrm{RNE}_{\text {all pairs }} \\
\mathrm{RNE}_{\text {pooled }}\end{array}$ & $\begin{array}{l}-0.23 \\
-0.13 \\
-0.22 \\
-0.21 \\
-0.19 \\
-0.24\end{array}$ & $\begin{array}{l}-2.08 \\
-1.12 \\
-2.24 \\
-2.31 \\
-1.64 \\
-1.62\end{array}$ & $\begin{array}{l}0.056 \\
0.280 \\
0.042 \\
0.037 \\
0.122 \\
0.128\end{array}$ & $\begin{array}{l}0.0019 \\
0.0015 \\
0.0019 \\
0.0019 \\
0.0017 \\
0.0022\end{array}$ & $\begin{array}{l}3.22 \\
2.48 \\
3.71 \\
3.90 \\
2.93 \\
2.87\end{array}$ & $\begin{array}{l}0.006 \\
0.027 \\
0.002 \\
0.002 \\
0.011 \\
0.012\end{array}$ & $\begin{array}{l}0.43 \\
0.30 \\
0.5 \\
0.52 \\
0.38 \\
0.37\end{array}$ & $\begin{array}{r}10.36 \\
6.14 \\
13.74 \\
15.19 \\
8.59 \\
8.24\end{array}$ \\
\hline $\begin{array}{l}\ln R R_{\text {min }} \\
\ln R R_{25} \text { pairs } \\
\operatorname{lnRR}_{50} \text { pairs } \\
L R_{100} R_{10 i r s} \\
\operatorname{lnRR}_{\text {all pairs }} \\
\ln R R_{\text {pooled }}\end{array}$ & $\begin{array}{l}-0.31 \\
-0.16 \\
-0.37 \\
-0.32 \\
-0.28 \\
-0.33\end{array}$ & $\begin{array}{l}-1.32 \\
-0.70 \\
-1.70 \\
-1.57 \\
-1.22 \\
-1.64\end{array}$ & $\begin{array}{l}0.206 \\
0.493 \\
0.112 \\
0.138 \\
0.241 \\
0.124\end{array}$ & $\begin{array}{l}0.0030 \\
0.0024 \\
0.0033 \\
0.0031 \\
0.0029 \\
0.0031\end{array}$ & $\begin{array}{l}2.44 \\
1.96 \\
2.93 \\
2.89 \\
2.38 \\
2.89\end{array}$ & $\begin{array}{l}0.029 \\
0.071 \\
0.011 \\
0.012 \\
0.032 \\
0.012\end{array}$ & $\begin{array}{l}0.30 \\
0.21 \\
0.38 \\
0.37 \\
0.29 \\
0.37\end{array}$ & $\begin{array}{l}5.96 \\
3.83 \\
8.59 \\
8.36 \\
5.65 \\
8.36\end{array}$ \\
\hline $\begin{array}{l}\mathrm{CRCI}_{\text {min }} \\
\mathrm{CRCI}_{25 \text { pairs }} \\
\mathrm{CRCI}_{50 \text { pairs }} \\
\mathrm{CRCI}_{100 \text { pairs }} \\
\mathrm{CRCI}_{\text {all pairs }} \\
\mathrm{CRCI}_{\text {pooled }}\end{array}$ & $\begin{array}{l}-0.24 \\
-0.13 \\
-0.24 \\
-0.23 \\
-0.20 \\
-0.25\end{array}$ & $\begin{array}{l}-1.88 \\
-1.01 \\
-2.08 \\
-2.09 \\
-1.53 \\
-1.62\end{array}$ & $\begin{array}{l}0.082 \\
0.330 \\
0.057 \\
0.055 \\
0.149 \\
0.128\end{array}$ & $\begin{array}{l}0.0020 \\
0.0016 \\
0.0022 \\
0.0021 \\
0.0019 \\
0.0023\end{array}$ & $\begin{array}{l}3.02 \\
2.34 \\
3.48 \\
3.60 \\
2.78 \\
2.87\end{array}$ & $\begin{array}{l}0.009 \\
0.035 \\
0.004 \\
0.003 \\
0.015 \\
0.012\end{array}$ & $\begin{array}{l}0.39 \\
0.28 \\
0.46 \\
0.48 \\
0.36 \\
0.37\end{array}$ & $\begin{array}{r}9.10 \\
5.47 \\
12.09 \\
12.96 \\
7.71 \\
8.26\end{array}$ \\
\hline
\end{tabular}

The two other currently used indices $-\ln R R$ and RNE - perform more reasonably. Moreover, the lnRR index is statistically sound and has a clear mathematical meaning. However, ecologists using $\ln R \mathrm{R}$ as an index of the intensity of competition (or facilitation) should recall that the arithmetic mean of logarithms is equal to the logarithm of the geometric mean. Patterns in lnRR thus reflect the combined effect of intensity and the spatial variability of competition (or facilitation) between plants, and tests for linear relationships in the index amount to tests of exponential patterns in relationships between plants. We see no indications of awareness of these issues in the analyses based on $\ln R \mathrm{R}$ (Hedges et al. 1999).

The RNE index corrects the worst bias of RCI and behaves reasonably when the intensity of competition or facilitation ranges from weak to moderate $(-0.5<$ $\mathrm{RNE}<+0.5$ ), as was the case in the study of Callaway et al. (2002). However, an index should be so designed that it can reflect differences in the processes of interest even when their intensity is high. The standard way to linearize a bounded index is arc sin transformation. This has little impact on index values within the ranges where RNE behaves reasonably, which facilitates comparisons between studies where RNE and CRCI has been used.

When a traditionally used index performs well, it is normally unadvisable to propose a new one. In this case, however, we feel that launching a new index is motivated. Ecologists tend to think in terms of arithmetic means and linear patterns - an opportunity that mathematically and statistically sound interpretation of the $\ln R R$ index does not provide. The other widely used index - RCI - has serious, built-in biases, which have been only partially corrected in the RNE index. There is thus a real need for an index, which reflects arithmetic means and linear patterns in plant-plant

Table 2. Overall mean values of coefficients and their difference from $0(\mathrm{~N}=16)$.

\begin{tabular}{lccc}
\hline Coefficient & value & $\mathrm{t}_{15}$ & $\mathrm{p}$ \\
\hline $\mathrm{RCI}_{\text {min }}$ & -0.33 & -1.93 & 0.073 \\
$\mathrm{RCI}_{25}$ pairs & -0.26 & -1.72 & 0.106 \\
$\mathrm{RCI}_{50 \text { pairs }}$ & -0.31 & -1.89 & 0.078 \\
$\mathrm{RCI}_{100 \text { pairs }}$ & -0.26 & -2.03 & 0.061 \\
$\mathrm{RCI}_{\text {all pairs }}$ & -0.29 & -2.03 & 0.061 \\
$\mathrm{RCI}_{\text {pooled }}$ & 0.14 & 1.83 & 0.088 \\
$\mathrm{RNE}_{\text {min }}$ & 0.10 & 1.84 & 0.086 \\
$\mathrm{RNE}_{25}$ pairs & 0.13 & 2.63 & 0.019 \\
$\mathrm{RNE}_{50}$ pairs & 0.12 & 2.29 & 0.037 \\
$\mathrm{RNE}_{100}$ pairs & 0.12 & 2.43 & 0.028 \\
$\mathrm{RNE}_{\text {all pairs }}$ & 0.12 & 2.27 & 0.038 \\
$\mathrm{RNE}_{\text {pooled }}$ & 0.15 & 2.23 & 0.042 \\
$\operatorname{lnRR}_{\text {min }}$ & 0.22 & 2.11 & 0.052 \\
$\operatorname{lnRR}_{25}$ pairs & 0.25 & 2.65 & 0.018 \\
$\operatorname{lnRR}_{50}$ pairs & 0.22 & 2.16 & 0.047 \\
lnRR $_{100}$ pairs & 0.22 & 2.36 & 0.032 \\
$\operatorname{lnRR}_{\text {all pairs }}$ & 0.23 & 2.23 & 0.042 \\
$\operatorname{lnRR}_{\text {pooled }}$ & 0.21 & 2.22 & 0.042 \\
$\mathrm{CRCI}_{\text {min }}$ & 0.12 & 1.92 & 0.073 \\
$\mathrm{CRCI}_{25}$ pairs & 0.15 & 2.65 & 0.018 \\
$\mathrm{CRCI}_{50}$ pairs & 0.13 & 2.26 & 0.039 \\
$\mathrm{CRCI}_{100}$ pairs & 0.13 & 2.41 & 0.029 \\
$\mathrm{CRCI}_{\text {all pairs }}$ & 0.14 & 2.26 & 0.039 \\
$\mathrm{CRCI}_{\text {pooled }}$ & 0.16 & 2.23 & 0.041 \\
\hline
\end{tabular}


interactions and which does not become biased when computed from pair-wise comparisons between neighbor removal and control plants. The CRCI index proposed here has these properties.

Acknowledgements - The study was supported by a grant from Royal Academy of Science (KVA) for collaboration between Sweden and countries that emerged from the former Soviet Union, a grant from Swedish Council for Natural Sciences (NFR), Swedish Council for Agriculture and Forestry Research (SJFR), and Academy of Finland, and by a grant no. 6048 from Estonian Science Foundation.

\section{References}

Aarssen, L. W. and Epp, G. A. 1990. Neighbour manipulations in natural vegetation: a review. - J. Veg. Sci. 1: 13-30.

Cahill, J. F. Jr. 1999. Fertilization effects on interactions between above- and belowground competition in an old field. - Ecology 80: 466-480.

Cahill, J. F. Jr. 2002. Interactions between root and shoot competition vary among species. - Oikos 99: 101-112.

Callaway, R. M., Brooker, R. W., Choler, P. et al. 2002. Positive interactions among alpine plants increase with stress. - Nature 417: 844-848.
Goldberg, D. and Scheiner, S. M. 1993. ANOVA and ANCOVA: field competition experiments. - In: Scheiner, S. M. and Gurevitch, J. (eds), Design and analysis of ecological experiments. Chapman and Hall, pp. 69-91.

Goldberg, D. E., Rajaniemi, T., Gurevitch, J. et al. 1999. Empirical approaches to quantifying interaction intensity: competition and facilitation along productivity gradients. - Ecology 80: 1118-1131.

Gough, L., Goldberg, D. E., Hershock, C. et al. 2001. Investigating the community consequences of competition among clonal plants. - Evol. Ecol. 15: 547-563.

Grace, J. B. 1995. On the measurement of plant competition intensity. - Ecology 76: 305-308.

Hedges, L. V., Gurevitch, J. and Curtis, P. 1999. The meta-analysis of response ratios in experimental ecology. - Ecology 80: 1150-1156.

Markham, J. H. and Chanway, C. P. 1996. Measuring plant neighbour effects. - Funct. Ecol. 10: 548-549.

Reader, R. J., Wilson, S. D., Belcher, J. W. et al. 1994. Plant competition in relation to neighbor biomass: an intercontinental study with Poa pratensis. - Ecology 75: 17531760.

Sammul, M., Oksanen, L. and Grünthal, M. 2005. Regional effects on competition-productivity realtionship: a set of field experiments in two distant regions. - Oikos 112: $138-148$

Wilson, S. D. and Tilman, D. 1993. Plant competition and resource availability in response to disturbance and fertilization. - Ecology 74: 599-611.

Subject Editor: Per Lundberg 